\title{
tic\&société
}

Vol. 3, $n^{\circ}$ 1-2| 2009

TIC et diasporas

\section{Internet et la reconfiguration de l'espace public tunisien : le rôle de la diaspora}

Romain Lecomte

\section{(2) OpenEdition \\ Journals}

Édition électronique

URL : https://journals.openedition.org/ticetsociete/702

DOI : $10.4000 /$ ticetsociete.702

Éditeur

Association ARTIC

\section{Référence électronique}

Romain Lecomte, «Internet et la reconfiguration de l'espace public tunisien : le rôle de la diaspora », tic\&société [En ligne], Vol. 3, n 1-2 | 2009, mis en ligne le 12 janvier 2010, consulté le 21 septembre 2021. URL : http://journals.openedition.org/ticetsociete/702 ; DOI : https://doi.org/10.4000/ ticetsociete.702 


\title{
Internet et la reconfiguration de l'espace public tunisien: le rôle de la diaspora
}

\author{
Romain Lecomte \\ Doctorant en sciences politiques et sociales \\ Service de Sociologie des nouvelles dynamiques sociales \\ Institut des Sciences humaines et sociales \\ Université de Liège \\ Boulevard du Rectorat, 7 \\ Bât. 31 \\ 4000 Liège, Belgique \\ Romain.lecomte@ulg.ac.be
}

Sociologue de formation, Romain Lecomte mène depuis deux années une thèse de doctorat à l'Université de Liège (Belgique) sur les nouvelles formes d'engagement et de critique émergeant au sein du cyberespace tunisien. Ses domaines d'intérêt principaux concernent l'impact des NTIC sur le lien social (étude des communautés virtuelles) et sur l'espace public. Dans le cadre de son DEA interuniversitaire (Université libre de Bruxelles, Université catholique de Louvain et Facultés universitaires de Saint-Louis), il a ainsi réalisé un mémoire sur les usages dissidents d'internet dans divers contextes autoritaires. Son intérêt porte également sur la situation politique tunisienne et, parallèlement à sa thèse de doctorat, il a conduit une enquête sur le congrès d'un parti d'opposition tunisien (Ettajdid) 


\title{
Internet et la reconfiguration de l'espace public tunisien : le rôle de la diaspora
}

\begin{abstract}
Résumé
Dans une approche sociologique, nous montrons comment des Tunisiens de la diaspora profitent de l'internet pour s'impliquer, s'informer et dénoncer ce qu'ils considèrent comme des injustices dans leur pays d'origine. Nous décrivons le style de discours critique privilégié par ces internautes militants, ainsi que le type d'espace public qu'ils revendiquent. Nous nous penchons sur le parcours militant de certains d'entre eux et analysons les rapports qu'ils entretiennent avec les internautes résidant en Tunisie, dans un premier temps, surtout, moins enclins à dénoncer ouvertement (recours à la critique indirecte) le régime autoritaire. Nous montrons cependant comment ces internautes en Tunisie vont jouer un rôle de plus en important au sein de ce nouvel espace contestataire.
\end{abstract}

Mots-clés : Internet, politique, cybermilitantisme, analyse de sites internet, espace public.

\begin{abstract}
Using a sociological approach, we show how Tunisians of the diaspora take advantage of Internet to keep themselves involved and informed and to denounce what they consider as unjust in their native country. We describe the style of critical speech preferred by these activist Internet users and the type of public sphere they claim. We study the activist paths of some of them and analyse their relationships with Internet users living in Tunisia. While they are initially less likely to openly denounce (use of indirect criticism) the authoritarian regime, we illustrate how these Internet users in Tunisia come to play an increasingly important role in this new space of contestation.
\end{abstract}

Keywords : Internet, politics, cyberactivism, website analysis, public sphere.

\section{Resumen}

Desde una perspectiva sociológica, se muestra cómo los Tunecinos de la diáspora sacan provecho de Internet para informarse, implicarse e incluso denunciar aquello que consideran como injusticias en su país de origen. Se describe el estilo del discurso privilegiado por estos internautas militantes, así como el tipo de espacio público que reivindican. Se analizan los 
Internet et la reconfiguration de l'espace public tunisien : le rôle de la diaspora

recorridos militantes de algunos de ellos y se estudian las relaciones que estos mantienen con los internautas residentes en Túnez. Estos, en un primer momento, son menos propensos a denunciar abiertamente al régimen autoritario (utilizan la crítica indirecta). Finalmente se muestra cómo estos internautas residentes en Túnez van a jugar un papel cada vez más importante en el seno de este nuevo espacio contestatario.

Palabras clave : Internet, política, cibermilitantismo, análisis de los sitios de Internet, espacio público. 
Romain LECOMTE

\section{Introduction}

Dans le contexte autoritaire tunisien, où les médias " traditionnels » que sont la télévision hertzienne et la presse ${ }^{1}$, sont muselés, on voit aujourd'hui émerger de nouvelles voix discordantes au sein d'un espace virtuel particulièrement difficile à contrôler. Parmi les Tunisiens qui ont décidé de profiter de l'avènement de l'internet pour contester le régime autoritaire du président Zine el-Abidine Ben Ali, nous verrons que ceux de la diaspora ont joué, en particulier durant les premières années, un rôle très important. Nous montrerons aussi que, progressivement, et non sans quelques conflits, les internautes tunisiens résidant au pays vont être de plus en plus actifs et acquérir une visibilité considérable à travers la blogosphère.

Pour mener notre analyse, nous nous basons sur des observations en ligne, des échanges par courriel et des entretiens semi-directifs avec des internautes tunisiens, résidant en Tunisie et à l'étranger ${ }^{2}$. L'approche que nous préconisons est une approche du «politique par le bas » (Bayart, 1992): nous nous intéressons aux marges du politique, à des acteurs souvent ignorés à la fois de l'analyse politique et des professionnels du politique. II s'agit ainsi, comme le préconisent Françoise Massit-Folléa et Cécile Méadel, « de ne plus considérer la pratique politique comme l'apanage des partis et organismes officiels, et de prendre en considération ce qui se fait "hors les murs", pour reprendre les termes de Bernard Manin » (2007, p.11).

\section{Genèse de la contestation en ligne en Tunisie : des sites et des forums}

Dans un premier temps, les protestations ouvertes à l'encontre du pouvoir tunisien se sont principalement développées au sein de quelques forums et sites, souvent qualifiés par les internautes tunisiens de « cyberdissidents ». Les membres de la diaspora tunisienne ont joué un rôle considérable dans la construction de cet espace virtuel contestataire. Dans cet article, lorsque nous parlerons de "cyberdissidence », nous ferons référence à des internautes tunisiens qui, d'une part, contestent de façon très ouverte, directe, le régime politique tunisien (pour ce qui est de la critique en ligne plus modérée et

\footnotetext{
${ }^{1}$ II existe trois journaux légalisés en Tunisie qui proposent un contenu réellement critique vis-à-vis du pouvoir en place. Cependant, ces journaux sont mal distribués et connaissent une faible diffusion.

${ }^{2}$ Une quarantaine d'entretiens et une dizaine d'échanges par courriel.
}

tic\&société - 3 (1-2), 2009 
Internet et la reconfiguration de l'espace public tunisien : le rôle de la diaspora

indirecte, cf. infra) et, d'autre part, n'appartiennent pas à des organisations politiques ou militantes ${ }^{3}$, du moins n'y jouent pas un rôle de premier plan ${ }^{4}$. Comme nous le verrons plus loin plus en détail, cette non appartenance a notamment pour corollaire une pratique et un langage contestataires distincts.

On peut situer la naissance de la " cyberdissidence » tunisienne en janvier 1998, lorsque deux étudiants tunisiens, Fœtus et Waterman, décident de créer une liste de diffusion appelée Takriz, mot arabe pouvant être traduit par « ras-lebol » et "emmerdement ». Deux ans plus tard, les deux fondateurs, ainsi que les membres les plus actifs de la liste font de Takriz un " e-mag », suscitant l'enthousiasme au sein de la jeunesse tunisienne. Ce site, qui cessera ses activités en 2002, traite dans un style mordant et sans révérence des sujets tabous en Tunisie tels que la religion, le sexe et la politique. Malgré la censure, il est d'après ses membres visité 2000 fois par jour ${ }^{5}$. Parmi les vingt-deux membres permanents de Takriz, plusieurs résident à l'étranger: "Nous sommes tous des jeunes Tunisiens (sauf un) qui en avons ras le bol du manque d'espace d'expression dans le pays. Nous sommes presque tous étudiants en Tunisie ou à l'étranger ${ }^{6}$. »

Dans la droite ligne de Takriz, Zouhair Yahyaoui (en Tunisie) fonde le site et forum Tunezine ${ }^{7}$ en juillet 2001, où des membres de la diaspora tunisienne vont également jouer un rôle de premier ordre. La plume satirique de Zouhair Yahyaoui, connu sous le pseudonyme Ettounsi, lui vaut d'être emprisonné en juillet 2002. Libéré en novembre 2003, affaibli après des conditions de détention difficiles et plusieurs grèves de la faim, il décédera d'une crise cardiaque le 13 mars 2005, à l'âge de 37 ans. II est aujourd'hui perçu comme un symbole, parfois même comme un martyr de la cyberdissidence par de nombreux internautes tunisiens, qui lui rendent régulièrement hommage. En 2003, il est

\footnotetext{
3 Ils n'appartiennent pas à ce que Michel Camau et Vincent Geisser appellent « l'espace [protestataire] multi-organisationnel plus ou moins autonome [..., où les identités politiques, partisanes et associatives ne sont jamais clairement tranchées et sont même parfois interchangeables » (2003, p.264). Pour parler de cet espace protestataire, nous utiliserons le terme " opposition classique », terme parfois utilisé par les internautes eux-mêmes.

${ }^{4}$ Cependant, il faut préciser que l'usage du terme « cyberdissident » que nous faisons ici n'est qu'un usage parmi d'autres que nous avons pu rencontrer. En effet, si les internautes font référence dans de nombreuses discussions en ligne à la "cyberdissidence » pour désigner exclusivement ces espaces indépendants vis-à-vis de l'opposition classique, il arrive également qu'eux-mêmes utilisent le mot " cyberdissident » pour désigner toute personne ayant été sanctionnée par les autorités tunisiennes pour des activités réalisées en ligne (parfois simplement pour avoir téléchargé des documents sur l'internet).

5 http://tempsreel.nouvelobs.com/actualites/international/20010821.OBS7638, consulté le 29 juin 2009.

Entretien de Martin Jouanneau avec un des membres, Don Quichotte: http://membres.lycos.fr/tuniscom/sept 2000.htm, consulté le 29 juin 2009.

http://www.tunezine.com
} 
désigné lauréat du premier prix «Cyberliberté » de «Reporters s@ns frontières ».

Le site RéveilTunisien (RT) est, lui, créé en 2002: «À la base ce site s'adossait sur Tunezine. Tunezine étant les forums et $R T$ le journal plus sérieux. Le sérieux perdurera dans la démarche mais le ton changera rapidement pour retrouver l'insolence de ton qui est la nôtre » (Hasni, par courriel, 2007). Ce site, fonctionnant comme un magazine électronique avec possibilité pour chacun d'écrire des commentaires ou proposer des articles, est durant tout un temps en grande partie géré par des non Tunisiens (déjà présents sur Tunezine). Ainsi, Angelica n'est pas Tunisienne (et réside à l'étranger), Sophie Piekarec, la fiancée de Zouhair Yahyaoui qui a repris la gestion de Tunezine après son arrestation et qui, de ce fait, est proche de RéveilTunisien, n'est pas non plus tunisienne. De même, Luiza Toscane, qui publie alors régulièrement sur $R T$, est une militante associative française. Cependant, là encore, l'un de ses membres les plus actifs (qui a, par la suite, géré seul le site) est un Tunisien vivant en France, Hasni. Quelques temps après la création du site, une Tunisienne au Canada, Houeida K. Anouar (Antekrista, déjà active sur Tunezine auparavant et ayant également « pris part à l'aventure Takriz »), viendra rejoindre l'équipe de $R T$.

L'internet est souvent vécu par nos interviewés comme un moyen de s'informer sur leur propre pays. Houeida K. Anouar, dès son arrivée dans son pays d'accueil, en 1998, a profité de l'accès à la fois plus développé et plus libre à l'internet pour s'informer sur la situation des droits de l'Homme en Tunisie :

\begin{abstract}
« Une fois au Canada, il y avait internet partout, tout était ouvert. [...] Pas de censure, c'était vraiment une sorte de feu d'artifice d'information. J'ai commencé à voir les dessous des choses, j'ai commencé à voir la Tunisie de l'extérieur à travers une autre lorgnette [...]. Je ne voyais plus la vision filtrée, du pays, de la sécurité... Je m'en doutais, ce n'était pas que j'ignorais ça mais je n'avais pas accès à l'information, avant. [...] J'ai fouiné, j'ai passé des nuits blanches sur internet à m'intéresser à ce qu'il se passait en Tunisie » (entretien en Tunisie, 2008).
\end{abstract}

Pour des raisons personnelles sans lien avec son activisme en ligne, Houeida K. Anouar est retournée en Tunisie en 2005 et y vit encore aujourd'hui. Profitant de sa présence au pays, elle a suivi de près le « Mouvement du 18 octobre », une grève de la faim menée par diverses personnalités de l'opposition en prévision du Sommet mondial sur la société de l'information (SMSI), qui s'est tenu à Tunis en novembre 2005. Membre du comité 
Internet et la reconfiguration de l'espace public tunisien : le rôle de la diaspora

d'information sur le mouvement, elle a joué en quelque sorte un rôle de relai avec la sphère virtuelle. Elle a notamment créé le blog de la grève de la faim ${ }^{8}$, censuré et piraté plusieurs fois. $\mathrm{Si}$, comme nous allons le voir plus loin, ces " cyberdissidents » ont souvent été critiques vis-à-vis de l'opposition tunisienne, ce genre d'initiative montre bien que des passerelles ont parfois pu être établies avec cette dernière.

Fondé en partie en réaction à la paire formée par RéveilTunisien et Tunezine, le site et forum Nawaat a été créé en avril 2004. II se présente comme $100 \%$ tunisien :

« II y avait un besoin de créer un site tunisien géré par des Tunisiens, et qui imposait une lignée éditoriale, sur les forums et sur l'espace général du site, de Tunisiens. Parce qu'en fait, sur Tunezine et RéveilTunisien, à un certain moment les administrateurs n'étaient pas tunisiens. [...] Hasni est tunisien, mais les autres non. Et là, imposer une certaine lignée sur ce qui se publie [...] sur le site était un peu délicat par rapport aux Tunisiens " (entretien en Hollande avec l'un des fondateurs du site, Sami Ben Gharbia, 2008) ${ }^{9}$.

Alors que Tunezine n'est plus en activité et que RéveilTunisien ne l'était plus jusqu'à sa réouverture récente (le site avait été hacké et détruit), Nawaat n'a cessé d'élargir sa visibilité et repenser son interface depuis sa création. Ses fondateurs et gestionnaires font partie de la diaspora tunisienne: Sami Ben Gharbia réside en Hollande, Malek Khadraoui (arrivé plus tard dans l'équipe de Nawaat) en France et Astrubal, Centrist et Mistral (désormais inactif sur le site) vivent également à l'étranger ${ }^{10}$. Tout comme Houeida K. Anouar, Malek Khadraoui souligne ce besoin de s'informer sur son pays natal qu'il venait de quitter :

« Je suffoquais un peu, on va dire, par le manque de liberté dans le pays. Donc je suis parti, et première chose quand on part dans un pays un peu plus libre [...], c'est d'essayer de se renseigner, d'avoir des

\footnotetext{
${ }^{8} \mathrm{http}: / /$ grevedelafaim.blogspot.com

9 Comme le souligne Hasni (échange par courriel, 2007, et entretien en France, 2009), un article de Luiza Toscane publié sur RéveilTunisien au sujet de l'antisémitisme en Tunisie a particulièrement suscité la polémique au sein du cyberespace contestataire tunisien.

${ }^{10}$ Par respect pour l'anonymat des personnes, nous serons parfois amené à ne pas mentionner certaines informations à leur sujet.
} 


\section{Romain LECOMTE}

infos, donc c'était le premier réflexe » (entretien en

France, 2008).

Il est lui aussi à Tunis au moment du «Mouvement du 18 octobre », ce qui lui permet de rencontrer les grévistes et, de façon moins officielle que Houeida K. Anouar, de servir de "lien entre le virtuel et le réel » (Malek Khadraoui, entretien cité). Sami Ben Gharbia a lui quitté la Tunisie pour des raisons politiques : portant un certain intérêt à la révolution islamique iranienne, il visite l'Iran à l'âge de 30 ans. Environ un an après son retour, il décide de s'exiler pour fuir une probable arrestation de la police tunisienne. Après un long périple, il arrive en Hollande en 1998. C'est en collectant des informations sur l'internet en vue de bénéficier du statut de réfugié politique (obtenu en 2004) que Sami Ben Gharbia commence à s'intéresser aux sites contestataires :

« J'ai commencé à collecter des informations sur la situation des libertés et des droits de l'homme en Tunisie, pour construire un dossier pour ma demande d'asile. Donc, c'était l'objectif, rassembler le plus de documents pour les présenter à mon avocate et au ministère de la Justice. [...] Et de là, j'ai fait la connaissance de ces sites dissidents, comme Takriz, les archives de Takriz et puis Tunezine et... je me suis investi petit à petit, en lisant et puis en commentant. $[\ldots]$ Je me suis engagé en tant que forumier ${ }^{11}[\ldots]$. Et après, j'ai pris le chemin de la cyberdissidence en construisant avec des amis [rencontrés au sein des espaces cyberdissidents] Nawaat et d'autres initiatives dissidentes » (entretien en Hollande, 2008).

Sami Ben Gharbia a fini par faire de son activisme en ligne son métier, travaillant désormais chez GlobalVoices, « organisation à but non-lucratif de blogueurs du monde entier » qui « a pour but de favoriser le dialogue mondial sur le Web, en présentant l'actualité de pays et de personnes souvent ignorés par les médias traditionnels ${ }^{12}$ ॥. Si certains de ces cybermilitants étaient déjà dotés de connaissances poussées en informatique, d'autres ont appris sur le terrain. Sami Ben Gharbia et Astrubal ont ainsi à la base une faible connaissance de l'informatique ou des nouvelles technologies de l'information et de la communication: " Je viens d'un milieu littéraire, j'ai fait littérature et un peu de droit, mais j'ai appris à manipuler l'ordinateur et l'internet sur le terrain,

\footnotetext{
11 Néologisme parfois utilisé par les internautes tunisiens, le terme "forumier » désigne un internaute très actif sur un ou des forums internet ( $y$ interagissant régulièrement et $y$ jouant éventuellement un rôle d'animation ou de modération).

12 http://fr.globalvoicesonline.org
}

tic\&société - 3 (1-2), 2009 
Internet et la reconfiguration de l'espace public tunisien : le rôle de la diaspora

donc je suis le fruit d'une expérience [militante] » (Sami Ben Gharbia, entretien) Pourtant, ces deux gestionnaires de Nawaat sont non seulement devenus des webmasters chevronnés, mais également des inventeurs d'outils informatiques pour mener leur activité militante. On peut notamment citer la carte des prisons tunisiennes de Sami Ben Gharbia (cf. infra) et deux logiciels, le générateur de langue de bois ${ }^{13}$ (outil humoristique consistant à générer un discours de propagande semblable à celui des journaux tunisiens) et le « 403 Access Denied Checker ${ }^{14}$ » (permettant de tester et vérifier la censure en Tunisie), mis au point par Astrubal.

À ce tableau de la première génération de sites contestataires tenus par des internautes sans appartenance politique, il faut également ajouter la liste de diffusion quotidienne Tunisnews $^{15}$. Créée en mai 2000 par cinq anonymes résidant à l'étranger qui seraient, "pour la plupart, des réfugiés politiques islamistes - des années $1990^{16}$ ", Tunisnews constitue une source d'information qui connaît un succès considérable en Tunisie et au-delà. Les responsables de Tunisnews, rassemblant des informations au contenu souvent politiquement " sensible » et provenant de sources très variées (communiqués de l'opposition, articles de la presse étrangère et des rares titres de la presse d'opposition Attariq Al Jadid, Al Mawkif, Mouatinoun -, articles de sites web et de blogs, etc.), correspondent à ce que Fabien Granjon appelle des militants-médiateurs de type filtreurs: "L'observation minutieuse des pratiques militantes de communication sur réseaux révèle [...] l'émergence d'une nouvelle classe d'intermédiaires, constituée de militants-médiateurs fortement investis dans des opérations de propagation de l'information » (2000, p.3). Et parmi les trois grandes catégories repérables d'intermédiaires, Granjon mentionne les " filtreurs ", qui ne se contentent pas de diffuser l'information, mais opèrent au préalable une sélection de l'information tendant à devenir pléthorique. "Les filtreurs s'assignent donc comme cadre d'exercice de soulager les militantsinternautes menacés par l'inflation des données » (2000, p.9). En effet, si les administrateurs de Tunisnews tiennent à publier quotidiennement « tous les communiqués, toutes les analyses, toutes les déclarations, toutes les prises de position, tous les articles et autres interventions indépendamment de la

\footnotetext{
${ }^{13}$ http://astrubal.nawaat.org/2004/04/20, consulté le 29 juin 2009.

14 http://astrubal.nawaat.org/403-access-denied-checker, consulté le 29 juin 2009.

${ }^{15}$ http://www.tunisnews.net/

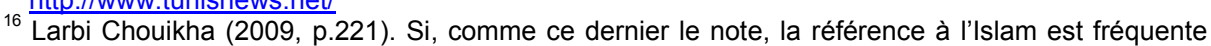
dans les textes écrits par les administrateurs de Tunisnews, ces derniers publient aussi des textes proposant des opinions très critiques vis-à-vis des islamistes, notamment du parti interdit Ennahda. Pour une analyse complète de Tunisnews, voir Larbi Chouikha (2009), qui précise également que la première liste de diffusion lancée par l'équipe de Tunisnews date d'octobre 1999 et se nommait Tunispress.
} 
personne, de l'organisation, du parti ou de la mouvance ${ }^{17}$ », ils prétendent le faire " après vérification " (ce qui n'empêche pas quelques fois la publication d'informations erronées) et sont nécessairement amenés à opérer une sélection lorsque, par exemple, ils choisissent de publier certains articles de blog plutôt que d'autres. En outre, ils opèrent une mise en forme. Enfin, de nombreux abonnés de la liste reprochent aux administrateurs de Tunisnews un filtrage idéologique : bien qu'ils publient des textes aux opinions variées, il semblerait qu'ils privilégient les sources islamistes ${ }^{18}$. Comme les autres sites cités plus haut, Tunisnews est diffusé depuis un serveur étranger (Suède).

Ces espaces en ligne n'ont bien entendu pas été les seuls où des Tunisiens « hors les murs » ont développé de nouvelles façons de s'opposer. D'autres espaces plus restreints, plus personnels, ont également existé, mais bien souvent gravitaient autour de ces sites et forums très fréquentés. Par ailleurs, des journalistes indépendants ont également lancé des sites critiques (notamment le site Alternatives Citoyennes de Nadia Omrane). Le fait que la diaspora ait constitué un élément pionnier de la résistance en ligne peut s'expliquer par divers facteurs. Tout d'abord, les Tunisiens vivant à l'étranger ont eu un accès à la fois plus développé et plus libre à l'internet. Ensuite, ils sont moins exposés à la répression que les Tunisiens en Tunisie, bien que de nombreux internautes tunisiens à l'étranger craignent que leurs activités contestataires en ligne causent des problèmes à leurs proches restés au pays ${ }^{19}$. Enfin, les expatriés tunisiens, du fait qu'ils résident généralement dans un pays démocratique, perçoivent souvent l'autoritarisme sévissant dans leur pays d'origine comme d'autant plus injuste et intolérable. Cependant, malgré ces conditions, déjà à cette époque, de jeunes Tunisiens ont pu participer - souvent de façon plus discrète - depuis la Tunisie à ce nouvel espace contestataire ${ }^{20}$.

\footnotetext{
17 Extrait de l'entretien «électronique » avec l'équipe de Tunisnews (publié dans Lamloum et Ravenel, 2002, pp.249-251). Dans cet entretien, les membres de Tunisnews expliquent aussi rester anonymes " afin d'assurer la sécurité de [leurs] familles et de [leurs] parents dans le pays ».

${ }^{18}$ Les administrateurs revendiquent cependant leur neutralité (voir l'entretien déjà cité avec l'équipe de Tunisnews dans Lamloum et Ravenel, 2002). Selon eux, cette abondance de textes islamistes sur Tunisnews serait le simple reflet de leur importante présence dans le cyberespace.

${ }^{19}$ Cette inquiétude, dont nous ont fait part plusieurs internautes résidant à l'étranger, s'explique par le fait que l'intimidation des membres de la famille et des proches d'opposants constitue une méthode répressive bien connue en Tunisie : chantage, pertes d'emploi ou d'aide sociale... Voir notamment Béatrice Hibou (2005) et le rapport de l'IFEX-TMG d'avril 2007.

${ }^{20}$ À ce titre, Takriz, à la différence de ses successeurs, présentait la particularité de comprendre de nombreux membres permanents en Tunisie. Autre spécificité, les membres les plus actifs y étaient majoritairement des étudiants, alors que ses successeurs comprendront davantage d'individus qui, tout en étant assez jeunes, étaient déjà engagés dans le monde professionnel.
}

tic\&société - 3 (1-2), 2009 


\section{Espace public alternatif et « anti-langue de bois »}

\subsection{Des critiques sans révérence et peu formalisées}

Dénonçant les nombreuses violations des droits de l'Homme commises en Tunisie, ces internautes revendiquent souvent par ailleurs leur indépendance vis-à-vis de « l'opposition » tunisienne, qu'il s'agisse de "l'opposition cliente », soutenant le pouvoir en place en échange de diverses rétributions matérielles et symboliques de la part de l'État, ou de «l'opposition indépendante », composée de quelques associations ou partis marginalisés. Affichant leur positionnement " hors les murs", ils s'expriment souvent de façon satirique et provocatrice, adoptant un style beaucoup plus informel que celui adopté par les personnalités de l'opposition tunisienne. Ces traits caractéristiques de la cyberdissidence sont résumés par Hasni (en France), administrateur de RéveilTunisien :

« La première [opposition] est l'officielle, celle qui appelle à voter Ben Ali. La seconde est la clandestine, peu contagieuse, souvent dogmatique et sans projet réel à proposer [...] et la troisième, c'était nous. Les internautes citoyens, les non encartés, les impertinents ne respectant pas leurs aînés, les incontrôlables qui ne demandent pas la permission et font ce qu'ils veulent avec les moyens du bord » (par courriel, 2007).

Zouhair Yahyaoui, le fondateur de Tunezine, présentait son site comme « indépendant de tout organe gouvernemental, de toute entité économique, de toute tendance politique ou croyance religieuse. [...] Tunezine est en fin de compte pitoyablement censuré, depuis sa création [...] mais les voies du netoyen $^{21}$ tunisien sont interminables » (sur Tunezine).

Takriz se définissait quant à lui comme le « premier e-magazine tunisien, $0 \%$ langue de bois ». De façon générale, ces cyber-résistants privilégient aujourd'hui encore, mais au sein d'espaces d'expression en ligne plus variés et dispersés - un style de langage informel, sans langue de bois et laissant une large place à la satire. Si la cible première des critiques de ces internautes contestataires sont les autorités tunisiennes au sens large, les partis politiques d'opposition ne sont eux-mêmes pas épargnés. Hasni parle ainsi d'une

\footnotetext{
${ }^{21}$ Francisation de «netizen », «netoyen » est un mot hybride (net et citoyen) désignant une personne s'exprimant sur la chose publique par le biais d'internet.
} 
opposition «souvent dogmatique et sans projet réel à proposer » (supra). DeZ estime "que l'opposition non reconnue ainsi que les personnalités et organisations de défense de droits de l'Homme ne fournissent que le strict minimum et communiquent très mal » (par courriel, 2007) :

« L'outil internet [... qui] est devenu et deviendra une arme de communication très efficace et de plus en plus redoutable est encore très mal utilisé par les structures "classiques". Les partis et personnalités politiques ont encore du mal à utiliser cet outil ou même à lui donner une importance relative. Ils [...] ne s'impliquent presque jamais sur les forums ou les blogs et ils ont encore beaucoup de réticences envers les sites et forums, parce qu'ils risquent d'être malmenés et bousculés par des citoyens certes et plus souvent anonymes mais très impliqués " (DeZ, sur son site ${ }^{22}$ et sur le forum Tunezine).

La prétention des cyberdissidents à pouvoir, "en tant que simples citoyens, critiquer les personnalités [de] la sphère publique » (Sami Ben Gharbia, sur Nawaat $^{23}$ ) a été régulièrement affichée: "Nous, en tant que forumiers et cyber-opposants, que ce soit de NT [Nawaat] ou de TZ [Tunezine] ou de n'importe quel espace de dissidence, devrions exiger des opposants de revoir leur stratégie » (Mkarriz, sur Tunezine ${ }^{24}$ ). Dans les débats au sein du forum Tunezine dans le cadre des "Conférences de Tunezine » (mai 2002), on lit bien cette volonté de démarcation vis-à-vis de l'opposition classique :

« II faut rester indépendants tant que l'on reprochera aux partis et à leur[s] dirigeant[s] de faire de la politique politicienne. Si nous soumettons quelque chose, il faut le faire à tous les partis et les inviter à réagir dans une tribune ouverte ${ }^{25}$. "

La chercheuse Célina Braun a même décrit Takriz comme un " anti parti »: "Son succès est révélateur de l'état d'esprit d'une partie de la jeune génération qui ne peut se reconnaître ni se faire encadrer par les partis politiques en présence ». Et, concernant le style de langage utilisé, elle souligne que «le contraste est fort avec le style des élites des partis ou des comités de défense des droits de l'Homme » (2006, pp.54-55). Outre ses "querelles intestines »,

\footnotetext{
${ }^{22}$ www.etunisie.net, aujourd'hui fermé.

23 http://www.nawaat.org/portail/2004/12/16, consulté le 29 juin 2009.

$24 \mathrm{http} / / /$ www.tunezine.com/article.php3?id article=759, consulté le 29 juin 2009.

25 http://www.reveiltunisien.org/spip.php?article36, consulté le 29 juin 2009.
} 
Internet et la reconfiguration de l'espace public tunisien : le rôle de la diaspora

son "dogmatisme », sa " politique politicienne », ces internautes ont souvent reproché à cette opposition son élitisme. Se faisant l'écho des critiques portées par de nombreux internautes, dans un article dressant le bilan du SMSI, Meryem Marzouki, chercheuse et responsable associative à Paris, écrit :

«Pourtant, toute cette jeunesse tunisienne qui participe aux forums de discussion sur internet, malgré les difficultés et les risques que cela comporte, qui fait preuve d'une créativité remarquable (voir tous les clips vidéos produits à l'occasion du SMSI, voir aussi la campagne "Yezzi" [cf. infra]), qui ne s'embarrasse pas de révérence, a-t-elle seulement été soutenue, encouragée, voire simplement mentionnée, par cette opposition [...] ? Bien au contraire, elle a été soigneusement écartée par des plus que cinquantenaires qui n'ont rien compris, sauf que cette jeunesse risquait décidément de bousculer leur traintrain » (Marzouki, 2005).

Ces différentes critiques rejoignent ce qu'ont pu dire les politologues Michel Camau et Vincent Geisser à propos de l'opposition tunisienne, soulignant « la multiplication des querelles et des dissensions » en son sein et expliquant que « l'incapacité des groupes proto-partisans à se constituer une base militante [...] ne tient pas exclusivement aux conditions objectives de la répression [...] mais aussi au sens profond qu'ils donnent à leur mission politique : conquérir, d'une part, un leadership purement symbolique sur un marché politique entièrement contrôlé par l'État et conforter, d'autre part, leur position d'extériorité par rapport aux masses "ignorantes" ". Les auteurs constatent également la « personnalisation du pouvoir et le faible renouvellement des dirigeants » (2003, pp.250 et 255).

\subsection{Un " contre-public subalterne "?}

La façon dont ces internautes contestataires de la "première génération » (précédant la multiplication des blogs courant 2006) définissent - actuellement encore, mais avec moins d'insistance - leurs pratiques et l'espace dissident qu'ils constituent, la façon aussi dont ils prennent leurs distances vis-à-vis de l'opposition classique, renvoient au concept de "sphère de contre-public » développé par Nancy Fraser (2003). Celle-ci, dans un article visant à repenser la sphère publique théorisée par Jürgen Habermas à partir du modèle bourgeois 


\section{Romain LECOMTE}

du XVIII ${ }^{\mathrm{e}}$ siècle $(1978 \text { [1962] })^{26}$, conteste sa vision d'une sphère publique unifiée, élitiste puisque composée exclusivement d'hommes blancs et propriétaires et propose au contraire de prendre en compte la multiplicité des sphères publiques. Fraser parle de ces contre-publics qui ont «contesté les normes exclusives du public bourgeois [...], élaborant de nouveaux styles de comportement politiques et de nouvelles normes de discours public " (Fraser, 2003, p.111). Elle appelle ces publics alternatifs " contre-publics subalternes dans le sens où ils forment des arènes discursives parallèles dans lesquelles les membres des groupes sociaux subordonnés élaborent des contre-discours, ce qui leur permet de fournir leur propre interprétation de leurs identités, de leurs intérêts et de leurs besoins » (Fraser, 2003, p.119). Cette idée de contre-public apparaît ainsi dans la revendication des cyberdissidents à constituer un public alternatif à l'opposition classique, ainsi que dans le type de discours moins formalisé et plus satirique qu'ils privilégient.

Dans un texte à la fois révélateur des opinions émises au sein des espaces " cyberdissidents" et du style de langage préconisé, écrit lors des "Conférences de Tunezine » (cf. infra), T.I.Z (très actif sur Tunezine et RT) explicite cette idée d'espace alternatif :

« Ils [les partis d'opposition] ont bien trop à faire [...] à dépasser leur propre caricature en s'engluant dans leur éternel jeu schizophrénique et suicidaire [...]. Le régime despotique a contribué à construire une opposition mal organisée, en manque d'inspiration. [...] II nous reste néanmoins une autre alternative. [...] il faut profiter des derniers espaces libres que nous détenons et qui nous sont offerts par les moyens technologiques, à savoir internet. II nous revient de prendre notre destin en main et d'impliquer toute la société dans un débat qui concerne son avenir. Et la meilleure fenêtre ouverte vers tous les citoyens reste la communication. Alors à nous d'exploiter les ressources internet: TUNeZINE, RéveilTunisien, TUNISNEWS pour lancer le débat. Le choix de ces supports n'est pas anodin, on a là un magazine

\footnotetext{
${ }^{26}$ Précisons que, comme Nancy Fraser le note elle-même, Jürgen Habermas a par la suite revu sa conception de la sphère publique, parlant désormais d'une pluralité d'« espaces publics partiels " à la fois distincts et entrelacés (Habermas, 1992, pp.161-191, et 1997). Pour une réflexion et une synthèse de travaux qui ont été menés sur l'espace public en général et l'espace public sur l'internet en particulier (en contexte démocratique et en contexte autoritaire), voir Romain Lecomte (2007).
}

tic\&société - 3 (1-2), 2009 
Internet et la reconfiguration de l'espace public tunisien : le rôle de la diaspora

électronique, un forum de débats libre, un site web indépendant et une liste de diffusion neutre ${ }^{27}$. »

Dans ces "Conférences de Tunezine », débats en ligne organisés en 2002, dont les synthèses figurent sur le site RéveilTunisien, des cyberdissidents tunisiens ont cherché à formaliser leurs revendications, leurs besoins, et notamment à réfléchir sur la façon dont ils pourraient à l'avenir collaborer avec l'opposition classique. Chaque internaute tunisien, s'il le souhaitait, pouvait formuler ses opinions et revendications et certains se sont chargés ensuite de synthétiser l'ensemble. II faut préciser qu'aujourd'hui, s'ils continuent à se poser en alternative, les cyberdissidents remettent plus rarement en cause les opposants classiques tunisiens.

Nancy Fraser souligne le caractère dual de ces contre-publics : d'une part, comme nous venons de le voir, " ils fonctionnent comme des espaces de repli et de regroupement », d'autre part, « ils fonctionnent aussi comme des bases et des terrains d'essais pour des activités d'agitation dirigées vers des publics plus larges » (2003, p.120). Si les cyberdissidents peinent parfois à se faire entendre auprès de publics plus larges (auprès de la population tunisienne dans son ensemble, mais aussi auprès des " opposants classiques »), ils sont néanmoins parvenus à réaliser quelques "coups " médiatiques, surtout auprès de publics étrangers. L'exemple le plus significatif à cet égard est la manifestation en ligne "Yezzi fock ! » : chaque individu souhaitant participer à la manifestation mettait sur le site internet consacré à la manifestation une photo de lui accompagné du slogan « Yezzi ! » (" Ça suffit ! »), écrit par exemple sur une pancarte tenue à la main ou ajouté sur la photo à l'aide d'un logiciel de retouche. Certains, afin de rester anonymes, dissimulaient leur visage sur la photo. Beaucoup de personnes, en Europe occidentale surtout, mais aussi en Tunisie, ont participé ou au moins pris connaissance de cette action collective qui a été rapportée par de nombreux médias étrangers (tels CNN ou Al Jazeera) ${ }^{28}$.

Cependant, ces internautes, s'ils ont souvent critiqué l'élitisme des opposants tunisiens, appartiennent eux-mêmes à une certaine élite, généralement dotée d'un accès à l'internet à domicile, résidant bien souvent dans la capitale (Tunis) ou dans un pays développé (France, Canada et ÉtatsUnis surtout). Cette remarque vaut également pour les blogueurs, dont nous allons parler plus loin. Si l'internet permet donc à des "profanes» de la politique de participer à des débats publics, on ne peut pour autant parler d'un espace public " populaire ». Les chaînes satellitaires, qui sont venues - avant l'internet - briser le monopole de l'État sur l'audiovisuel, si elles ne permettent que très peu aux «profanes » de la politique de participer réellement au débat

\footnotetext{
${ }^{27} \mathrm{http}: / /$ www.reveiltunisien.org/spip.php?article607, consulté le 29 juin 2009.

28 http://www.yezzi.org/press.php
} 
public, présentent par contre l'avantage d'être plus largement accessibles. En effet, malgré une législation visant à contrôler de façon plus stricte les usages des antennes paraboliques et d'autres stratégies visant à lutter contre «la concurrence des chaînes étrangères, [l'État] alternant répression, offensives diplomatiques et séductions par imitation des formats télévisuels transnationaux ", n'a pu contenir la réception croissante des chaînes satellitaires et l'intérêt « des Tunisiens pour une information dissonante » (Ferjani, 2002, p.175).

Comme le souligne Peter Dahlgren, dans un article envisageant l'application du concept d'espace public à l'internet dans le contexte des démocraties occidentales, "le profil sociologique des usagers du Net est à ce jour bien délimité : il se caractérise par un biais important en faveur d'hommes jeunes, riches et dotés d'un fort capital culturel » (Dahlgren, 2000, p.174). Cette fracture numérique contribue ainsi à une sphère publique exclusive, élitiste. Sur ce point, elle n'est donc pas tellement différente de la sphère publique bourgeoise du $\mathrm{XVIII}$ siècle mise en avant par Jürgen Habermas, que Nancy Fraser et d'autres ont critiqué. Or, cette fracture est encore plus prononcée dans un pays en voie de développement comme la Tunisie. Si on peut observer une nette progression de l'accès à l'internet ces dernières années, celui-ci reste néanmoins restreint à une petite frange de la population ${ }^{29}$. En avril 2009, le nombre officiel d'« utilisateurs " s'élève à un peu moins de trois millions (soit un taux de pénétration d'environ $29 \%$ ), mais le nombre d'« abonnés ", lui, reste limité à environ 300000 (soit environ $3 \%$ de la population) ${ }^{30}$. Or, comme nous l'ont dit pratiquement tous les internautes rencontrés, il est difficile de bloguer ou d'interagir régulièrement sur des forums sans avoir un accès régulier à l'internet.

\section{Conflit entre cyberdissidents et internautes modérés}

Jusqu'il y a peu, on pouvait observer au sein de l'internet tunisien deux catégories distinctes d'internautes: d'une part, les "cyberdissidents ", dénonçant de façon permanente et directe les autorités tunisiennes (à commencer par le président Ben Ali et son entourage), d'autre part, les

\footnotetext{
29 Tant dans le discours officiel que dans les politiques publiques adoptées, on peut observer une réelle volonté de la part du gouvernement d'accroître cet accès à l'internet (Bras, 2007, p.162). Cependant, ces mesures ont eu jusqu'à maintenant une portée limitée, du fait notamment d'une gestion économique et politique très interventionniste, "s'inscrivant dans le paradoxe de la modernisation autoritaire, où l'État se mobilise en faveur de la diffusion des TIC, mais avec des modes de mobilisation qui entravent celle-ci » (Bras, 2007, p.179).

${ }^{30}$ http://www.ati.tn Voir aussi les statistiques de I'UIT : http://www.itu.int
}

tic\&société - 3 (1-2), 2009 
Internet et la reconfiguration de l'espace public tunisien : le rôle de la diaspora

internautes tunisiens n'abordant pas ou peu de thèmes connotés politiquement. Bien que ces deux catégories d'internautes interagissaient très peu au sein de l'espace virtuel, lorsque cela arrivait, cela donnait parfois lieu à des disputes très animées. C'est au sein de la "blogosphère " tunisienne que nous avons pu observer ces interactions.

Les cyberdissidents ont pendant plusieurs mois dénoncé l'apolitisme de la majorité des blogueurs tunisiens, acceptant docilement la « dictature ». C'est en décembre 2005 que nous repérons les premières grandes tensions autour de la question de l'implication citoyenne de la blogosphère tunisienne. Sami Ben Gharbia, alors l'un des rares cybermilitants à tenir un blog, publie un article comparant les blogs tunisiens à " des zones touristiques »: "Elles s'installent loin de la misère locale, l'évitent et la contournent ». Sami Ben Gharbia a écrit ce texte dénonçant le caractère "politiquement correct » d'une grande partie des blogs tunisiens en réaction au refus de Houssein (informaticien au Canada), webmaster de Tn-blogs, l'agrégateur des blogs tunisiens le plus populaire ${ }^{31}$, d'indexer son blog:

"Cette affaire dépasse mon cas personnel puisque je n'étais pas le seul à me voir refuser l'entrée à ce club privé tunisien [...]. Le fait qu'ils [les blogueurs qui ne sont pas ou plus indexés dans l'agrégateur] remplissent les pages de leur blog par des textes et des pensées hostiles à la dictature tunisienne suffit pour les sanctionner aux yeux des responsables de cet annuaire indexant les seuls blogs d'une supposée jeunesse libre qui forment le bataillon des blogueurs adeptes du « politiquement correct. » [...] Cette vision du blogging divise la communauté des blogueurs tunisiens en deux mondes distincts [...] » (Sami Ben Gharbia, sur son $\operatorname{blog}^{32}$ )

Le texte de Sami Ben Gharbia suscite un large débat au sein de la blogosphère (avec de nombreux commentaires et articles sur la question). Et le

\footnotetext{
${ }^{31}$ Cet agrégateur Tn-blogs fonctionne comme une sorte d'annuaire, un site centralisateur qui référence une grande partie des blogs tunisiens et informe des derniers articles parus sur ceux-ci. II joue un rôle important dans l'interactivité entre les différents blogueurs. Pour repérer les blogs, lire les premières lignes des nouveaux articles publiés quotidiennement, saisir de façon globale les grands sujets de discussion abordés au sein des blogs tunisiens à un moment donné, cet agrégateur nous a été d'une grande utilité. Outre Tn-blogs (http://tn-blogs.com), le premier agrégateur tunisien, on peut également citer Tuniblogs (http://tuniblogs.com) et Taht Essour (http://taht-essour.com), ce dernier étant plus sélectif.

${ }^{32}$ http://www.kitab.nl/2005/12/20, consulté le 29 juin 2009.
} 


\section{Romain LECOMTE}

débat va redoubler d'intensité neuf mois plus tard, en septembre 2006. Suite au faible écho reçu par «la carte des prisons tunisiennes " (cf. infra) auprès des blogueurs tunisiens, alors que cette même action est largement médiatisée au sein d'espaces non tunisiens, Astrubal, Sami Ben Gharbia et quelques autres internautes très contestataires dénoncent de nouveau "l'apolitisme " des blogueurs tunisiens, Astrubal allant jusqu'à surnommer très péjorativement la blogosphère tunisienne par le qualificatif suivant : «lobotomisphère ${ }^{33}$ ".

Un grand nombre de blogueurs vont réagir à ces critiques. Houssein admet publiquement avoir refusé le blog de Sami Ben Gharbia parce qu'il était trop politisé. II admet également avoir agi par peur : " Je suis, comme beaucoup de blogueurs de tn-blogs, [comme ces] jeunes [...] déchirés entre cette envie de liberté et une peur maladive, inculquée par un système que tu connais très bien. [...] Je salue ton courage et tous ceux qui militent à visage découvert. Moi je ne l'ai pas. » (Commentaire sur le blog de Sami Ben Gharbia, décembre 2005.) On voit ici comment l'administrateur du site est contraint de participer bien malgré lui à une censure de l'internet s'opérant à divers niveau $x^{34}$. Cependant, il refuse de porter la responsabilité de cet isolement de quelques «blogueurs dissidents " et dénonce, comme d'autres blogueurs, le mépris affiché par les cyberdissidents :

"L'agrégateur n'est pas la cause de l'isolement des quelques blogs "cyberdissidents". [...] Si les blogueurs tunisiens forment une communauté endogène, la communauté dissidente, elle, est une entité close, opaque, et auto satisfaite. [...] Le militantisme virtuel reste confiné à quelques forums et webzines bien connus, tous inaccessibles en Tunisie. [...] À ces anonymes des forums, [...] qui jugent sans savoir, du haut de leur piédestal numérique, si supérieurs à la masse vendue et ignorante, si méprisants de leurs concitoyens, je vous dis que ce n'est pas avec pareille attitude que vous ferez évoluer les mentalités et changer les choses » (sur son blog, septembre 2006).

\footnotetext{
${ }^{33}$ Sur le forum aujourd'hui fermé de Nawaat et commentaire sur le blog de Sami Ben Gharbia, http://www.kitab.nl/2006/09/24, consulté le 29 juin 2009.

${ }^{34}$ La censure de l'internet en Tunisie, si elle est orchestrée par l'Agence tunisienne de l'internet (ATI) à un niveau national, s'opère à de multiples niveaux: fournisseurs d'accès, gérants de " publinets » (cybercafés), administrateurs de forum (il est généralement demandé, dans la charte des forums tunisiens, de ne pas parler de la politique nationale), simples blogueurs effaçant des commentaires "sensibles" postés par d'autres sur leur blog, etc. Ce système de censure pyramidale découle du fait que la responsabilité d'actions ou d'écrits interdits pèse sur un grand nombre d'acteurs.
}

tic\&société - 3 (1-2), 2009 
Internet et la reconfiguration de l'espace public tunisien : le rôle de la diaspora

De nombreux blogueurs tunisiens réagissent en dénonçant, comme Houssein, l'élitisme et le mépris affichés par ces cyberdissidents et en revendiquant une façon plus modérée et progressive de s'intéresser à la chose publique. C'est ce que fait Nadia From Tunis, Iorsqu'elle prône un " élargissement progressif et réfléchi de notre marge de manœuvre " et interroge : "La société serait-elle stratifiée, avec les cyberdissidents sur la plus haute marche, sorte de noblesse patriotique en charge de la conscience du peuple [...] ? » (sur son blog, novembre 2006) ${ }^{35}$. Les propos du blogueur Big Trap Boy (en Tunisie), plaidant aussi pour une critique plus graduelle et moins frontale, illustrent par ailleurs l'importance que peut revêtir le fait de résider en Tunisie ou à l'étranger en ce qui concerne le type de critique utilisée :

« Je ne vois pas pourquoi un bloggeur vivant en Tunisie irait se créer des problèmes ou faire bloquer l'accès à son blog juste pour faire plaisir à ces Che Guevara virtuels vivant à l'étranger. Ceci dit, moi personnellement je parle de politique dans mon blog, mais je n'essaie pas de me faire passer pour un militant ou de chercher la polémique, je crois qu'il faut faire évoluer les choses graduellement, c'est mieux que de chercher le conflit en permanence " (commentaire de Big Trap Boy sur le blog d'Infinity, septembre 2006).

$\mathrm{Si}$, comme l'indique Big Trap Boy, le lieu de résidence peut parfois expliquer le caractère modéré ou radical de la contestation en ligne et si, parmi les internautes les plus contestataires, figurent beaucoup de Tunisiens à l'étranger, il convient toutefois de nuancer: ces conflits n'opposent pas simplement Tunisiens en Tunisie et Tunisiens à l'étranger. Parmi les blogueurs modérés ayant participé à ces débats, certains se trouvent à l'étranger. C'est le cas de Houssein, au Canada depuis 1999. Si, faute de place, nous ne pouvons analyser ici en profondeur les diverses raisons qui peuvent pousser un Tunisien à l'étranger à n'émettre que des opinions très modérées sur la " cité » tunisienne, rappelons que le souci de protéger les proches encore au pays et de pouvoir lui-même y retourner quand bon lui semble, constitue un facteur important.

\footnotetext{
${ }^{35}$ Cette blogueuse, qui vivait alors en Tunisie, est partie ensuite en France, dans le courant de l'année 2007 (par courriel, 2007).
} 


\section{Romain LECOMTE}

Ainsi, ces tensions survenues fin 2005 et en septembre 2006 entre des cyberdissidents ${ }^{36}$ et de nombreux blogueurs tunisiens modérés révèlent alors une profonde division entre ces deux catégories d'acteurs. De façon générale, ils s'accordent pour déplorer l'existence d'" un monde entre les blogueurs tunisiens et la dissidence ${ }^{37}$ », mais divergent sur les causes de cette division.

De nouvelles tensions apparaissent fin 2006 entre ces deux catégories d'internautes, après que de nombreux blogueurs ont participé à l'action « Note blanche ", organisée pour la première fois le 25 décembre 2006, et reproduite à la même date en 2007 et 2008 . Cette action collective est révélatrice à la fois de l'amplification de la contestation de la part des blogueurs tunisiens qui s'amorce alors (cf. infra) et, par les débats auxquels elle donne lieu, des tensions entre internautes militants et non militants, tensions qui seront de moins en moins visibles par la suite. Elle consiste à poster un article vide le 25 décembre ou comprenant simplement le logo de la manifestation. Elle est initiée la première fois par solidarité pour trois blogueurs - Felsfa, Sami III (tous deux en Tunisie) et Samsoun ${ }^{38}$ (aux États-Unis) - tunisiens non «militants » venant d'être censurés. Si de nombreux blogueurs participent alors à cette action, c'est que, comme les trois blogueurs censurés eux-mêmes, ils n'estiment pas qu'il s'agit de « blogs dissidents » dépassant les « lignes rouges ».

Plusieurs cyberdissidents tunisiens se réjouissent de la solidarité affichée par de nombreux blogueurs vis-à-vis de cette censure, ainsi que des articles critiques qui l'ont provoquée. Ils y voient le signe d'une nouvelle étape pour la blogosphère tunisienne. Les blogueurs non militants, et notamment deux des trois blogueurs censurés, manifestent, eux, leur refus de voir la "Note blanche » être « instrumentalisée » par la cyberdissidence et considérée comme un acte politique. Ainsi, Sami III, constatant qu'il reçoit de plus en plus de visites d'internautes ayant transité par des sites dissidents censurés (il cite notamment Tunisnews et Nawaat) et ayant lu les textes enthousiastes des cybermilitants sur cette vague de contestation touchant la blogosphère tunisienne, s'insurge :

« Je vous demande de ne pas modifier mes dires et y extraire les "morceaux" qui vous servent! Moi je ne parle dans mon blog, que de ma vie et ce qui m'entoure, ET PUISQUE JE N'AI AUCUNE ACTIVITÉ

\footnotetext{
${ }^{36} \mathrm{Si}$ seuls quelques cyberdissidents ont participé directement à ces débats, on remarque que bien souvent, les internautes modérés ont réagi en critiquant les « cyberdissidents » dans leur ensemble. ${ }^{37}$ Commentaire de Adibs, septembre 2005, sur le blog de Houssein.

38 Leurs blogs sont respectivement: http://felsfa.hautetfort.com, http://sami-iii.blogspot.com, http://samsoum-usa.blogspot.com. Des trois blogueurs, seul Samsoun n'a pas effacé de son blog les articles pouvant être jugés subversifs. Au contraire, il a continué par la suite à intervenir sur des questions « sensibles ». Le fait qu'il soit le seul des trois à ne pas vivre en Tunisie a probablement joué dans cette décision.
}

tic\&société - 3 (1-2), 2009 
Internet et la reconfiguration de l'espace public tunisien : le rôle de la diaspora

\author{
POLITIQUE, JE NE PARLE JAMAIS DE \\ POLITIQUE !!!! PAS DE POLITIQUE ICI !!!! [...] Mon \\ action du lundi, si j'y participe, c'est pour que je puisse \\ parler de ce que je veux, pas de ce que vous \\ voulez ! » (Sami III, sur son blog) ${ }^{39}$.
}

Et Felsfa, dont le blog a également été censuré, d'aller dans le même sens en décidant même de ne pas participer à l'action collective pourtant organisée pour le soutenir :

« [...] Je crie haut et fort aussi que c'est malhonnête de pousser les gens à choisir un camp via de slogans attractifs avec des arrières pensées qui ne sont pas déclarées. Attention, vous allez poster blanc, ou montrer votre solidarité ce lundi. Vous allez faire une action qui sera qualifiée, par ceux qui l'ont cherchée, comme un " mouvement " vers je ne sais pas quoi. [...] ÉVITEZ le piège. Felsfa ne postera pas blanc ${ }^{40} »$.

\title{
4. Émergence d'une blogosphère citoyenne : le « tournant tunisien »?
}

Depuis cette époque, le paysage de la "cyber-citoyenneté » tunisienne a fortement évolué. Alors que nous avions observé, fin 2005 et fin 2006, des discussions très conflictuelles entre blogueurs modérés et internautes très contestataires, ces tensions ont aujourd'hui disparu au profit d'un engagement commun (mais comportant des degrés variables de contestation) dans les débats citoyens. En effet, en concomitance avec l'augmentation du nombre de blogs, de plus en plus de blogueurs (dont beaucoup sont en Tunisie), s'ils refusent généralement l'étiquette d'« opposants", de "dissidents" ou de " militants », s'expriment régulièrement de façon critique sur la chose publique. Un échange de plus en plus intense va ainsi s'établir entre Tunisiens en Tunisie et Tunisiens à l'étranger, ces derniers profitant notamment d'un accès développé et libre aux médias internationaux (en ligne et hors ligne) pour appuyer leurs contributions (écrits, dessins, vidéos...), les premiers développant une critique souvent plus ancrée dans leur quotidien (anecdotes, humour typiquement tunisien, photos prises ici et là...). Un va-et-vient s'opère ainsi, les

\footnotetext{
${ }^{39}$ http://sami-iii-int.blogspot.com/2006/12/chres-lecteurs.html, consulté le 29 juin 2009.

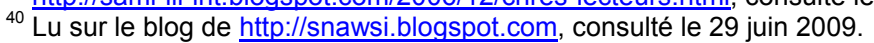




\section{Romain LECOMTE}

uns et les autres s'informant mutuellement au sein d'un même espace. Chez de nombreux blogueurs, une certaine prudence demeure cependant de mise (bien que de plus en plus rarement) quant aux cibles de la critique (ils évitent de remettre directement en cause les plus hauts responsables de l'État, le PartiÉtat, l'entourage du président, de parler de la torture dans les prisons...) et au langage utilisé. Partiellement en lien avec cette prudence, l'humour et la satire, déjà présents au sein des sites et forums très contestataires étudiés ${ }^{41}$, sont devenus un vecteur davantage privilégié encore de la contestation. Progressivement, la blogosphère est apparue comme l'espace dominant de la contestation en ligne ${ }^{42}$. Plus récemment, ce vent de contestation a également touché le site de réseau social Facebook.

Certains journaux ou revues tunisiens ont d'ailleurs commencé à s'intéresser aux propos émis par les blogueurs (tantôt pour en proposer une vision positive, tantôt au contraire pour les critiquer). C'est le cas de la rare presse d'opposition ${ }^{43}$, mais aussi de la presse modérée et de la presse progouvernementale. Le fait que la presse traditionnelle s'intéresse de plus en plus à ce qui se dit au sein de la blogosphère tunisienne, contribue à un décloisonnement (certes, encore très relatif) de cet espace contestataire. De même, du 23 au 25 mai 2008, le MEPI (Middle East Partnership Initiative) et I'IREX (International Research \& Exchanges Board), respectivement organisation gérée par le Département d'Etat américain et ONG internationale, ont invité une dizaine de blogueurs tunisiens ${ }^{44}$, résidant en Tunisie et à l'étranger (France, Canada, Hollande), à venir débattre sur le journalisme citoyen à Casablanca avec d'autres blogueurs nord-africains.

Pour mieux comprendre les " arts de faire", les "tactiques" mises en œuvre par ces blogueurs tunisiens afin de s'aménager des espaces d'autonomie pour résister aux pouvoirs en place et dénoncer ceux qui les servent (à commencer par les médias « traditionnels » tunisiens), nous allons faire référence ici aux travaux de James $C$. Scott. Celui-ci étudie les formes d'expression du mécontentement qui se déploient en secret ou de façon déguisée, tout en restant souvent dans les limites de l'acceptable, prenant en

\footnotetext{
${ }^{41}$ En 2002, Larbi Chouikha soulignait déjà à propos de la contestation en ligne en Tunisie : «Ces nouvelles formes d'information portent à la fois sur des modes d'expression, mais aussi de contestation. [...] Elles [...] rejoignent des formes de communication traditionnelles ancrées dans notre tradition orale. Ces anciennes formes déjouent la censure ou le manque d'information dans les noukat (anecdotes et dérision politique) et les rumeurs les plus extravagantes qui prennent généralement pour cibles les dignitaires de l'État » (2002, p.207).

${ }_{42}^{2}$ Le dernier rapport de Freedom House (2009) estime à 600 le nombre de blogs tunisiens actifs.

${ }^{43}$ Le journal d'Ettajdid, Attariq Al Jadid, publie par exemple la rubrique hebdomadaire « À travers la blogosphère ", comprenant que les blogs tunisiens sont devenus peu à peu une composante du paysage médiatique tunisien avec laquelle les partis politiques doivent désormais compter (entretien avec Baccar Gherib, militant de Ettajdid et journaliste à Attariq Al Jadid, Tunisie, 2009)

${ }^{44}$ Six de ces blogueurs ont été rencontrés : quatre en Tunisie et deux à l'étranger.
}

tic\&société - 3 (1-2), 2009 
Internet et la reconfiguration de l'espace public tunisien : le rôle de la diaspora

compte les "lignes rouges " comme les blogueurs tunisiens les appellent souvent. Les discours anti-hégémoniques auxquels il s'intéresse sont développés au sein de sites sociaux spécifiques qui, précise-t-il, ne sont pas simplement des espaces laissés vides par les dominants, mais sont gagnés, aménagés, construits et défendus par ceux qui résistent: une lutte s'engage pour s'approprier de tels espaces (Scott, 1990, p.123). Ainsi, dans le cas tunisien, les espaces virtuels où se diffusent les discours contestataires sont soumis à la surveillance et la censure de la "police de l'internet », dont l'instance principale est l'ATI ${ }^{45}$. Pour échapper à la censure ou à la répression, les internautes tunisiens déploient une multitude de tactiques de contournement et de camouflage (techniques et langagières). Scott parle d'«arts du déguisement politique », qui consistent en des "stratégies multiples par lesquelles des groupes subordonnés parviennent à insinuer la résistance, dans des formes déguisées, dans le "public transcript" » (Scott, 1990, p.136). Par "public transcript», il faut comprendre les pratiques et discours qui sont exposés aux regards des dominants, qui ne sont pas complètement " privés » ou « cachés".

Avant d'évoquer les formes déguisées de critique, il convient de nous attarder brièvement sur les ruses proprement techniques ${ }^{46}$. La méthode de contournement de la censure la plus connue est le proxy, serveur intermédiaire installé entre l'ordinateur de l'utilisateur et l'internet, lui permettant ainsi d'accéder à des sites web censurés dans son pays, en passant par une adresse IP autre que la sienne (localisée à l'étranger). Nous avons pu induire de nos observations et entretiens que beaucoup d'internautes tunisiens connaissent parfaitement l'usage des proxys et sont en mesure de contourner la censure. Cela ne veut pas dire pour autant que beaucoup d'entre eux y ont régulièrement recours, le fait même que ces sites soient censurés et donc interdits constituant un facteur dissuasif. Depuis que la critique s'est largement diffusée au sein de nouveaux espaces, le recours aux proxys semble cependant être chose de plus en plus courante.

Au sein des sites et forums contestataires de la première génération, où les formes de protestation s'avéraient souvent très directes, les messages déguisés, voilés, étaient plus rares. Les tortures dans les prisons tunisiennes, la prévarication des grandes familles dans l'entourage présidentiel, par exemple, étaient souvent explicitement abordées. Par contre, l'anonymat constituait la

\footnotetext{
${ }^{45}$ Voir Jean-Philippe Bras (2003). Le boycott de l'édition 2008 des « Tunisian Blog Awards » illustre bien également cette lutte des blogueurs pour l'appropriation et la protection de ce nouvel espace critique. Cf. http://fr.globalvoicesonline.org/2008/12/15, consulté le 29 juin 2009.

${ }^{46}$ Nous nous attardons peu dans cet article sur les aspects techniques de la censure et de son contournement. Ces aspects mériteraient un article à eux-seuls. Concernant la censure en Tunisie, voir notamment l'étude de l'Open Net Initiative (2005) et l'article de Nart Villeneuve (sur la Tunisie et d'autres pays) (2006).
} 


\section{Romain LECOMTE}

norme. Au sein de la blogosphère tunisienne, on remarque que les messages critiques sont généralement davantage euphémisé ${ }^{47}$, déguisés sous forme d'allusions, d'humour, de dessins, de métaphores... Nadia From Tunis explique ainsi en quoi ces blogs " citoyens " diffèrent des sites ou blogs "dissidents " : "Nous utilisons des ruses d'écriture et plus de subtilité pour passer à travers les filtres et ne pas nous faire repérer pour un seul post et perdre ainsi nos blogs » (par courriel, 2007). II s'agit notamment d'éviter certains mots-clés susceptibles d'attirer les censeurs, tels que «Ben Ali ».

La ruse, au sein des blogs tunisiens, va souvent de pair avec l'humour. Le blog Normalland, par exemple, avec une large dose d'humour et de satire, constitue une caricature de la Tunisie, imaginant un pays fictif, un leader dictatorial et diverses positions gouvernementales attribuées à d'autres blogueurs. Parmi les départements gouvernementaux, certains sont consacrés au marché noir et à la corruption ${ }^{48}$.

La critique s'insinue aussi dans des allusions, des anecdotes, des récits d'expériences personnelles. Plus haut, Sami III, pour insister sur le caractère non politique de son blog, affirmait : « Moi je ne parle dans mon blog, que de ma vie et ce qui m'entoure ». Or, il arrive fréquemment que des blogueurs en Tunisie parlent de leur vie personnelle tout en critiquant le système. C'est le cas lorsque Montassar (entretien en Tunisie, 2009) raconte sur son blog qu'après que l'un de ses collègues de travail ait ramené au bureau le drapeau tunisien, d'autres collègues l'ont taquiné : "C'est quoi, c'est une nouvelle "chooba" [cellule du RCD, le parti au pouvoir ${ }^{49}$ ] que vous avez ouvert ici ${ }^{50}$ ? " Montassar commente cette blague :

« Je ne sais pas pourquoi, mais moi aussi, j'avais la même impression, ça m'a fait bizarre de voir un drapeau aussi énorme aux locaux de l'entreprise, je l'avais moi même taquiné, le pauvre, il a été obligé de rappeler à chaque fois qu'il s'agit du drapeau du pays et non pas du RCD. Je ne vais pas en faire un drame, mais au fond, ça m'a fait de la peine que le drapeau ait été intuitivement associé à la "chooba" plutôt qu'au pays... » (sur son blog).

\footnotetext{
${ }^{47}$ Les euphémismes sont aussi un moyen de « jauger » continuellement la frontière langagière de ce qui est permissible aux yeux des détenteurs du pouvoir. Voir Scott (1990, pp.152-153). ${ }_{48}^{48}$ http://ounormal.blogspot.com

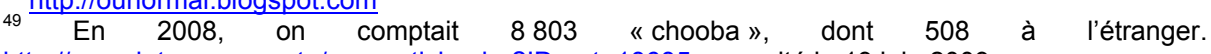
http://www.letemps.com.tn/pop article.php?ID art=18685, consulté le 19 juin 2009. ${ }^{50}$ http://m0ntassar.blogspot.com/2008/01/le-drapeau-de-la-tunisie-pas-de-Ircd.html, consulté le 29 juin 2009.
} 
Internet et la reconfiguration de l'espace public tunisien : le rôle de la diaspora

Par cette anecdote, Montassar témoigne (et les commentaires sur son blog vont aussi dans ce sens) de l'omniprésence du RCD, Parti-État de fait. Beaucoup de blogueurs tunisiens ont par ailleurs fait de nécessité vertu : le style indirect, codé, étant apprécié autant pour sa dimension esthétique et créative que pour sa dimension pratique (éviter la censure ou toute autre forme de répression). C'est notamment pour cette raison que le style d'écriture (en arabe littéraire et dialectal) de la blogueuse Arabicca, vivant en Tunisie et bloguant régulièrement depuis juillet 2007 (entretien en Tunisie, 2008), est très apprécié au sein de la blogosphère. Selon un autre blogueur, Carpe Diem, qui vit en France, Arabicca «a un style d'écriture très varié, très riche, elle utilise beaucoup d'images et, en fait, en [la] lisant [...], il faut lire entre les lignes, ce qui est un style très attrayant, finalement. Parce que ça fait travailler l'imagination, il y a beaucoup de "non dits" " (entretien en Tunisie, 2009). De même, le blogueur Big Trap Boy, vivant en Tunisie et bloguant depuis août 2006, s'est fait connaître par sa critique humoristique en dialecte tunisien.

Ces nouveaux blogs critiques sont souvent plus prudents quant à leurs cibles. Ainsi, si le gouvernement lui-même est encore rarement remis en cause explicitement, la presse tunisienne qui le porte aux nues fait par contre partie des cibles privilégiées de la critique. Le blog collectif Boudourou, créé en juillet 2007 et animé par quelques Tunisiens résidant presque tous en Tunisie ${ }^{51}$, qui analyse et se moque de cette presse, constitue un bon exemple de critique à la fois humoristique et relativement prudente quant à ses cibles. Ce blog passe au crible les journaux tunisiens, pro-gouvernementaux essentiellement, dénonçant les erreurs, les plagiats et la langue de bois qui y prédomine. Très ironiquement, des prix Boudourou («boudourou » désigne la pièce de monnaie tunisienne ayant la valeur la plus faible) d'or, d'argent et de bronze sont remis aux journalistes ayant écrit les articles les plus médiocres...

Parmi les autres cibles faisant régulièrement l'objet de critiques, figurent également en bonne place les grands projets de construction immobilière peu soucieux de l'environnement et du patrimoine. Sous l'impulsion de -Z-, les blogueurs se sont par exemple mobilisés, lançant en 2008 un groupe sur Facebook et une pétition pour la protection de l'île de la Zembra, menacée par un groupe d'investisseurs chinois souhaitant y implanter un grand complexe touristique.

James $\mathrm{C}$. Scott remarque à propos de ces formes déguisées et indirectes de contestation que l'alternative à l'anonymat, qui permet souvent de parler de façon " agressive », directe, aux dirigeants ${ }^{52}$, consiste à voiler le message juste

\footnotetext{
${ }^{51}$ http://boudourou.blogspot.com. Outre Free-Race, cité plus haut, nous avons rencontré un autre membre de Boudourou : Bel Malwene.

52 John Bohman (2004, p.138), comme Dale E. Eickelman et Jon W. Anderson (2003, p.8) soulignent le rôle important que peut jouer l'anonymat en ligne pour la constitution d'un espace
} 
suffisamment pour éviter les représailles. Et il ajoute: "L'essentiel de l'art verbal des groupes subordonnés consiste en des euphémismes astucieux qui, comme Zora Neale Hurston l'a noté, "sont caractérisés par un commentaire social et une critique indirects, voilés" » (Scott, 1990, p.153). II s'agit notamment de ne pas expliciter ses intentions contestataires, de telle manière que, même si elles sont comprises par les dominants, a posteriori, il soit toujours possible de les nier, de jouer sur l'ambiguïté. À ce sujet, Free-Race, l'un des blogueurs de Boudourou qui tient également un blog personnel, confie: "Les codes de langage et l'humour permettent de faire passer des messages codés, que même l'ATI comprend, même les agents comprennent, mais ils sont plus tolérants, soi-disant que peu de gens peuvent comprendre ce message ou... Donc, on emploie même des termes, par exemple «la famille » pour désigner les proches [du Président Ben Ali] ou le « patron » pour désigner le chef [Ben Ali], ainsi de suite » (entretien, Tunisie, 2009).

Face à un pouvoir autoritaire, répressif, les dominés ont donc, pour critiquer en public, au moins deux possibilités: déguiser le message ou déguiser le messager (anonymat). Dans le cas de la critique en ligne tunisienne, les deux formes de déguisement sont parfois utilisées simultanément.

Cependant, s'il est vrai que de nombreux blogueurs recourent à des ruses pour exprimer des opinions critiques vis-à-vis du système autoritaire, comme on l'a dit plus haut, cette prudence semble de moins en moins de mise. Cette prolifération des critiques s'est par ailleurs accompagnée de la censure de nombreux blogs. Outre l'action " Note blanche », dont nous avons déjà parlé, d'autres actions de solidarité ont été organisées pour contester cette censure et/ou revendiquer la liberté d'expression. Le $1^{\text {er }}$ juillet 2007, Samsoun avait ainsi lancé la journée « Je blogue pour la liberté d'expression ${ }^{53}$ ». Cette action, reproduite un an plus tard, a été largement suivie. Le 4 novembre 2008, les blogueurs ont lancé une «journée nationale pour la liberté d'expression » (également sur Facebook). Cette date a été choisie en solidarité avec Zied El Heni, le journaliste et blogueur tunisien qui a intenté un procès à l'ATI, accusant l'Agence d'avoir censuré Facebook (Zied El Heni, entretien en Tunisie, 2009). Pour marquer leur refus de la censure, les blogueurs ont aussi créé un blog collectif $^{54}$. "Ammar », personnification du censeur (également surnommé "Mkass », signifiant " ciseaux » en Tunisien) ou encore la célèbre page d'erreur 404 (s'affichant lorsqu'un site est censuré), constituent ainsi désormais

public. Toujours dans l'optique d'une réflexion sur l'espace public en ligne, Zizi Papacharissi synthétise les avantages et inconvénients de l'anonymat pour la constitution d'un « espace public virtuel » (2002).

${ }^{53}$ http://samsoum-us.blogspot.com/2007/06/le-1er-juillet-je-blogue-pour-la-libert.html, consulté le 29 juin 2009.

$\underline{\text { http://anticensuretounes.blogspot.com }}$

tic\&société - 3 (1-2), 2009 
Internet et la reconfiguration de l'espace public tunisien : le rôle de la diaspora

un sujet de discussion et d'indignation permanent au sein de la blogosphère tunisienne.

\section{Curiosité pour la chose publique et nouvelles technologies}

De façon générale, qu'il s'agisse des internautes investissant les sites et forums dissidents ou des nouveaux blogueurs, notre observation des pratiques et discours contestataires en ligne rejoint les remarques d'Arlette Farge (1992) sur l'espace public plébéien français du XVIII ${ }^{e}$ siècle. Tout comme la réflexion de Nancy Fraser sur les contre-publics, l'investigation d'Arlette Farge a été lancée en réaction à L'espace public (1978 [1962]), où Jürgen Habermas laissait consciemment de côté la variante plébéienne de la sphère publique parce que celle-ci était réprimée et apparaissait sans poids politique. Farge montre comment, à Paris, s'est développée une parole publique crainte par les autorités. L'auteur constate « l'avidité que montre la population parisienne pour se mettre au courant de ce qui survient». La cité se veut un "espace d'information où les habitants s'organisent [gazettes, nouvelles à la main, placards, caricatures sur les murs...] pour mieux savoir, pour défaire le secret tenu par le roi et la monarchie. La curiosité publique [est] un acte qui fait entrer chacun en politique" (1992, p.289). Et "ce goût pour l'information et les pratiques pour se l'approprier » s'expliquent notamment par l'attitude de la monarchie qui, tout en considérant la parole populaire comme vulgaire, la pourchasse et la réprime. Elle note aussi le caractère multiforme, difficile à saisir de cette parole populaire de plus en plus envahissante, au gré des évènements. Les observateurs de police sont les premiers à deviner « que l'opinion populaire, habituellement disqualifiée politiquement, [devient] un discours au sens politique évident » (Farge, 1992, p.45).

Cette description s'applique d'autant mieux aujourd'hui que la prolifération des blogs et Facebook viennent encore renforcer l'aspect multiforme et dispersé de cet espace virtuel contestataire de plus en plus difficile à contrôler pour les autorités. Au sein de la blogosphère, chaque blogueur " citoyen » construit son espace personnel à sa manière: certains privilégient la caricature, certains I'humour tunisien, certains la langue française ou même anglaise, certains la poésie... Ce qui permet à cet ensemble hétéroclite de blogs citoyens éparpillés de constituer un espace critique, c'est la multitude de liens hypertextes qui les relient, les commentaires que chacun peut poster suite à un article, mais aussi les quelques « agrégateurs » (cf. supra).

Ces internautes insistent régulièrement sur leur compétence et leur droit à critiquer. Et, en particulier dans le cas des cyberdissidents, à dénoncer les 
pratiques du «Roi ZABA », de la «Princesse Leila " ${ }^{55}$ et de "sa cour» (entourage du pouvoir, notamment les membres de la famille Trabelsi), mais aussi, comme on l'a montré, l'opposition classique elle-même. Comme dans l'espace public plébéien, la caricature est une forme de critique très présente sur ces espaces virtuels. L'internaute tunisien probablement le plus connu dans ce style de critique est -Z-, résidant à l'étranger et auteur du blog DEBATunisie ${ }^{56}$ (entretien, 2009). Dans ses dessins, -Z-, qui a commencé à bloguer en septembre 2007 et fait partie de ces nouveaux blogueurs intervenant régulièrement sur la chose publique, représente de façon critique et humoristique l'actualité nationale. II se moque régulièrement de la propagande officielle et de la mégalomanie présidentielle et dénonce «les mégaprojets [économiques] et le bradage du bien public ». Comme beaucoup d'autres internautes tunisiens, il fait régulièrement des allusions moqueuses à la couleur mauve, au chiffre 7 ou à la thématique du "Changement », qui sont autant d'éléments constitutifs de la symbolique du pouvoir: le président Ben Ali est arrivé au pouvoir le 7 novembre 1987, début de ce qu'il appelle depuis plus de 20 ans le « Changement »; la couleur mauve est sa couleur favorite, qu'il utilise notamment dans la décoration de ses slogans de campagne présidentielle. Les dessins de -Z- sont accompagnés de textes, qui se présentent souvent sous forme de fictions critiques.

Quant à la curiosité et la soif de savoir évoquées par Arlette Farge, elles font d'une certaine manière écho à l'avidité et la rapidité avec lesquelles ces internautes rapportent ce qu'il se passe en (et ce qui se dit sur la) Tunisie. Prenons ici comme exemples les actions des blogueurs cyberdissidents Astrubal et Sami Ben Gharbia. Astrubal a repéré et réuni toute une série de photographies datées (qui avaient été prises et mises en ligne par différents photographes amateurs d'avions) sur lesquelles figure l'avion présidentiel, et cela à différents endroits du globe où il n'était pas censé se trouver à la date indiquée. Astrubal a ensuite réalisé un montage (photos défilant, avec des commentaires écrits apparaissant sur les images et des cartes géographiques pour montrer pour chaque photo l'emplacement précis de l'avion) et pose des questions: "Qui utilise l'avion de la présidence de la République, lorsque ce n'est pas le chef de l'État ? Et à quoi cet avion, payé et entretenu par le contribuable tunisien, sert-il ? Qui contrôle l'utilisation "raisonnable" de cet outil $[\ldots]^{57}$ ? ॥ La « carte des prisons tunisiennes » constitue un autre bon exemple de cette curiosité pour la chose publique (cette volonté de dévoilement) associée aux nouvelles technologies: à partir notamment d'images satellites, Sami Ben Gharbia a élaboré une carte des prisons tunisiennes combinant

\footnotetext{
${ }^{55}$ Leila Ben Ali (dont le nom de jeune fille est Trabelsi).

${ }^{56}$ http://debatunisie.canalblog.com, consulté le 5 juillet 2009.

57 http://astrubal.nawaat.org/2007/08/29, consulté le 29 juin 2009.
} 
Internet et la reconfiguration de l'espace public tunisien : le rôle de la diaspora

diverses applications ${ }^{58}$, incluant des informations concernant les prisonniers politiques qu'elles contiennent, les raisons de leur incarcération...

\section{Conclusion}

Dans un contexte autoritaire peu propice à l'émergence d'espaces d'expression autonome, on a pourtant pu observer, au cours des dix dernières années, un espace virtuel de discussion et d'actions contestataires émerger et se diversifier tout en étant parfois traversé par des tensions internes. Au sein de cet espace, les Tunisiens vivant à l'étranger, jouissant d'un accès plus développé à l'internet et évoluant dans un contexte démocratique, ont joué dans un premier temps un rôle moteur, investissant surtout un nombre limité d'espaces collectifs (sites et forums). Progressivement, les Tunisiens demeurés au pays sont intervenus de plus en plus sur la chose publique, jouant un rôle primordial dans le développement de la blogosphère tunisienne. Parfois dans un style différent, avec leurs avantages respectifs, Tunisiens en Tunisie et Tunisiens à l'étranger continuent de développer cet espace contestataire en ligne, l'élargissant notamment à Facebook.

Si l'impact de ce nouvel espace d'expression autonome sur la société tunisienne, sur ses institutions, est actuellement pratiquement inexistant, il faut souligner que cette activité critique en ligne est encore récente et, comme nous l'avons montré, tend à s'élargir à de nouveaux acteurs et de nouveaux espaces depuis quelques années. II s'agit d'un espace d'expérimentation de débat public et d'activisme. Ce phénomène va probablement s'accentuer à l'avenir, au vu de la volonté réelle du gouvernement tunisien de généraliser l'accès à l'internet en Tunisie, démontrant ainsi son attitude ambivalente à l'égard de celui-ci : d'une part, il souhaite une démocratisation de l'internet - c'est-à-dire que davantage de Tunisiens y aient culturellement et matériellement accès - et d'autre part, il craint la possibilité d'une démocratisation par cet outil et exerce ainsi un sévère contrôle sur son utilisation ${ }^{59}$.

\footnotetext{
${ }^{58}$ http://www.kitab.nl/tunisianprisonersmap, consulté le 29 juin 2009.

59 Sur cette distinction entre "démocratisation du numérique " et "démocratisation [par le] numérique », voir Yves Gonzalez-Quijano (2004).
} 


\section{Références bibliographiques}

BAYART J.-F., 1992, La politique par le bas en Afrique noire : contribution à une problématique de la démocratie, Paris, Karthala.

BOHMAN J., 2004, "Expanding the dialogue: The Internet, the public sphere and prospects for transnational democracy ", dans CROSSLEY N. et J. M. ROBERTS (dir), After Habermas. New perspectives on the public sphere, Oxford, Blackwell Publishing, The Sociological Review, pp.131-155.

BRAUN C., 2006, "À quoi servent les partis tunisiens ? Sens et contre-sens d'une "libéralisation" politique ", Revue des mondes musulmans et de la Méditerranée, n¹11-112, pp.15-61.

BRAS J.-P., 2003, "Ordre public, politiques publiques et internet en Tunisie », dans F. MERMIER (dir), Mondialisation et nouveaux médias dans l'espace arabe, Paris, Maisonneuve \& Larose, pp.247-260.

BRAS J.-P., 2007, "Internet au Maroc et en Tunisie », dans M. MEZOUAGHI, Le Maghreb dans l'économie numérique, Paris, Maisonneuve \& Larose, pp.161-180.

CAMAU M. et GEISSER V., 2003, Le syndrome autoritaire. Politique en Tunisie de Bourguiba à Ben Ali, Paris, Presses de Sciences Po.

CHOUIKHA L., 2002, " Autoritarisme étatique et débrouillardise individuelle », dans O. LAMLOUM et B. RAVENEL (dir), La Tunisie de Ben Ali. La société contre le régime, Paris, L'Harmattan, pp.197-212.

CHOUIKHA L., 2009, " Un cyberspace autonome dans un espace autoritaire : l'expérience de Tunisnews ", dans K. MOHSEN-FINAN (dir), Les Médias en Méditerranée : nouveaux médias, monde arabe et relations internationales ", Arles, Actes Sud et MMSH.

DAHLGREN P., 2000, « L'espace public et l'internet », Réseaux, vol.18, n¹00, pp.159-186.

EICKELMAN D. F. et J. W. ANDERSON, 2003, "Redefining Muslim publics », dans D. F. EICKELMAN et J. W. ANDERSON (dir), New media in the Muslim world: The emerging public sphere, Bloomington, Indiana University Press, pp.1-18.

FARGE A., 1992, Dire et mal dire, Seuil, Paris. 
Internet et la reconfiguration de l'espace public tunisien : le rôle de la diaspora

FERJANI R., 2002, "Internationalisation du champ télévisuel en Tunisie », dans T. MATTELART, La mondialisation des médias contre la censure. Tiers Monde et audiovisuel sans frontières, Bruxelles, De Boeck, pp.155-175.

FRASER N., 2003, « Repenser l'espace public : une contribution à la critique de la démocratie réellement existante ", dans E. RENAULT et Y. SINTOMER (dir), Où en est la théorie critique?, Paris, La Découverte, pp.103-134.

FREEDOM HOUSE, 2009, Freedom on the Net: A global assessment of Internet and digital media, http://www.freedomhouse.org/template.cfm? page $=383 \&$ report $=79$, dernière consultation le 5 juillet 2009 .

GONZALEZ-QUIJANO Y., 2004, "À la recherche d'un internet arabe : démocratisation numérique ou démocratisation par le numérique?", Maghreb-Machrek, n 178, pp.11-29.

GRANJON F., 2000, "Les militants internautes : passeurs, filtreurs et interprètes », Multitudes, http://multitudes.samizdat.net/Les-militantsinternautes-passeurs, dernière consultation le 29 juin 2009.

HABERMAS J., 1978 [1ère éd. orig. 1962], L'espace public : archéologie de la publicité comme dimension constitutive de la société bourgeoise, Paris, Payot.

HABERMAS J., 1992, " "L'espace public", trente après », Quaderni, n¹8, pp.161-191.

HABERMAS J., 1997, Droit et Démocratie. Entre faits et normes, Paris, Gallimard.

HIBOU B., 2005, «Économie politique de la répression : le cas de la Tunisie », Raisons politiques, $\mathrm{n}^{\circ} 20$, pp.9-36.

IFEX-TMG, 2007, Le siège se poursuit, http://www.ifex.org/tunisia/2007/04/11/fourth tmg report launched worldwid e/fr/, dernière consultation le 5 juillet 2009.

LAMLOUM O. et B. RAVENEL (dir), 2002, La Tunisie de Ben Ali. La société contre le régime, Paris, L'Harmattan.

LECOMTE R., 2007, Réflexion sur la cyberdissidence et la sphère publique, Mémoire de DEA interuniversitaire en sciences sociales, Université Libre de Bruxelles, Université Catholique de Louvain, Facultés Universitaires de Saint-Louis.

MASSIT FOLLÉA et MÉADEL C., 2007, «Introduction : communication et débat public », Hermès, n47, pp.9-17. 
MARZOUKI M., 2005, "Pendant les travaux, le gâchis continue », Alternatives citoyennes, 24 novembre, $\mathrm{n}^{\circ} 17, \quad \mathrm{http}: / / \mathrm{www}$.alternativescitoyennes.sgdg.org/num17/dos-bilan-w.html, dernière consultation le 29 juin 2009.

OPEN NET INITIATIVE, "Internet Filtering in Tunisia in 2005: A Country Study ", http://opennet.net/studies/tunisia, dernière consultation le 29 juin 2009.

PAPACHARISSI Z, 2002, "The virtual sphere: the Internet as a public sphere ", New Media and Society, vol. 4, n¹, pp.9-27.

SCOTT J. C., 1990, Domination and the Arts of Resistance : Hidden Transcripts, New Haven, Yale University Press.

VILLENEUVE N., 2006, "The filtering matrix: Integrated mechanisms of information control and the demarcation of borders in cyberspace ", First Monday, http://www.firstmonday.org/issues/issue11 1/villeneuve/index.html, dernière consultation le 29 juin 2009. 